\title{
Neural network particle picking and denoising in cryoEM with Topaz
}

\author{
A Noble ${ }^{1}$, T Bepler $^{2}$, B Berger ${ }^{2}$ \\ ${ }^{1}$ New York Structural Biology Center / Simons Electron Microscopy Center, NEW YORK, NY, ${ }^{2}$ MIT, \\ Cambridge, $M A$ \\ anoble@nysbc.org
}

Single particle cryoEM projects are often hampered by low SNR particle views, which are missed by most particle pickers or severely de-prioritized causing junk to be preferentially picked. Moreover, for non-globular, small, asymmetric, and aggregated proteins, picking and centering such particles becomes critical. To solve these issues and more, we present Topaz particle picking using a novel positive-unlabelled framework and Topaz-Denoise using the Noise2Noise framework. We show that Topaz and Topaz-Denoise significantly increase the number of real particles picked, enable conventionally difficult projects, significantly decrease classification bias, and increase collection efficiency. We show the first in-depth analysis of pre-trained 2D and 3D denoising models for cryoEM and cryoET, which remove the characteristic sheets of noise in cryoEM micrographs of proteins and cryoET cellular tomograms. In the past few months, Topaz has been used by several labs around the world to enable and optimize single particle SARS-CoV2 projects. These recent projects and more will be highlighted to show the unique and timely power of Topaz. We will also highlight Topaz integration into the most popular cryoEM suites: Relion, CryoSPARC, Scipion, and Appion.

Acta Cryst. (2020). A76, a221 